\title{
Spectral Map Reconstruction Using Pan-Sharpening Algorithm: Enhancing Chemical Imaging with AFM-IR
}

\author{
Nikolay Borodinov*1, Natasha Bilkey ${ }^{2}$, Marcus Foston ${ }^{2}$, Anton V. Ievlev ${ }^{1}$, Alex Belianinov ${ }^{1}$, Stephen \\ Jesse $^{1}$, Rama K. Vasudevan ${ }^{1}$, Sergei V. Kalinin ${ }^{1}$ and Olga S. Ovchinnikova ${ }^{1}$ \\ 1. Center for Nanophase Materials Science, Oak Ridge National Laboratory, Oak Ridge, TN, USA. \\ 2. Department of Energy, Environmental \& Chemical Engineering, Washington University in St. Louis, \\ St. Louis, MO, USA. \\ * Corresponding author: ni1@ornl.gov
}

Recent advances in chemical imaging allow material composition characterization at the nanoscale. This significantly boosts the fundamental understanding of materials, detailed understanding of their structure, and guides the overall design of novel functional materials. Chemical imaging methods (optical spectroscopy, secondary ion spectrometry, mass spectrometry to name a few) vary in data acquisition, sample preparation, and spatial resolution; and thus, offer common yet nonidentical applicability for different samples [1]. However, many of these methods have a common feature - the chemical composition assignment is based on spectral characteristics acquired in a well-defined nanoscale region. As the field matures, more instruments and operational modes become available generating more data and requiring relevant processing tools used to operate on images, spectra, and combinations of thereof.

Coupling atomic force microscopy with infrared spectroscopy is a recent addition to the chemical imaging toolkit. A pulsed infrared laser triggers periodic thermal expansion of the sample, which is then detected by an AFM tip. As the absorption of the IR light is wavenumber-specific and distinct for different materials, it is possible to correlate regions of similarity with specific material or sample property. Recent advances in AFM-IR allow imaging of polymer blends and nanocomposites, biological tissue, and ion migration; which makes it a highly relevant technique [2]. Currently there are two operational modes: an acquisition of a single wavenumber map, which has full AFM resolution, and an acquisition of a sparse arrays at full spectral resolution. A direct attempt to measure full spectral response at each point of an AFM scan would be prohibitively long, and very likely to be disturbed by sample drift. In order to yield a full resolution dataset which could be used for correlative analysis, the two types of data - a spectral array and a set of single wavenumber maps, have to be combined in a physically meaningful way. Methods for combining multiple data channels with different spectral and spatial resolution have been intensively explored and overall are commonly referred to as pansharpening (Figure 1). One of these approaches, which is very suitable for the case of AFM-IR, relies on coupled non-negative matrix factorization (CNMF) of the data.

We demonstrate the applicability of CNMF-PS algorithm for synthetic data, experimental AFM-IR with known ground truth and an experimental AFM-IR with unknown ground truth. We analyze the algorithm parameters (downsampling rate, number of NMF components, number of single wavenumber maps used) on the quality metrics of the PS operation. We estimate the geometric and spectral distortion of the CNMF algorithm output for both experimental and synthetic data and yield practical recommendations on how to perform reconstruction of the AFM-IR datasets (Figure 2). We use our method for the analysis of plant cell wall materials and derive the correlation between the spatial distributions of chemically dissimilar components provided by CNMF-PS and local physical property 
(mechanical stiffness measured as the shift in AFM tip contact resonance). This example highlights the utility of PS algorithm for in-depth nanoscale characterization of various materials.

This work can be readily adopted by other chemical imaging techniques generating spectral images. It allows physics-based constraints on the data reconstruction process and results in full spectral and full spatial resolution maps which would be impossible or impractical to measure directly. CNMF-PS offers a novel outlook on the chemical imaging measurement output as a subset of a bigger dataset which can be efficiently reconstructed and used for further analysis of localized properties [3].

References:

[1] A Belianinov et al, ACS Nano 12 (12) (2018), p. 11798.

[2] A Dazzi and C B Prater, Chem. Rev. 117 (7) (2017), p. 5146.

[3] The authors acknowledge the Center for Nanophase Materials Sciences, which is a US DOE Office of Science User Facility supported under Contract DE-AC05-00OR22725, (N.B., A.V.I, A.B., S.V.K, O.S.O). A portion of algorithm development was a part of the AI Initiative, sponsored by the Laboratory Directed Research and Development Program of Oak Ridge National Laboratory (S.J., R.K.V), managed by UT-Battelle, LLC, for the U.S. Department of Energy (DOE). The plant sciences portion of this work was supported by the Center for Engineering MechanoBiology (CEMB), an NSF Science and Technology Center, under grant agreement CMMI: 15-48571 (N.B. and M.F.).

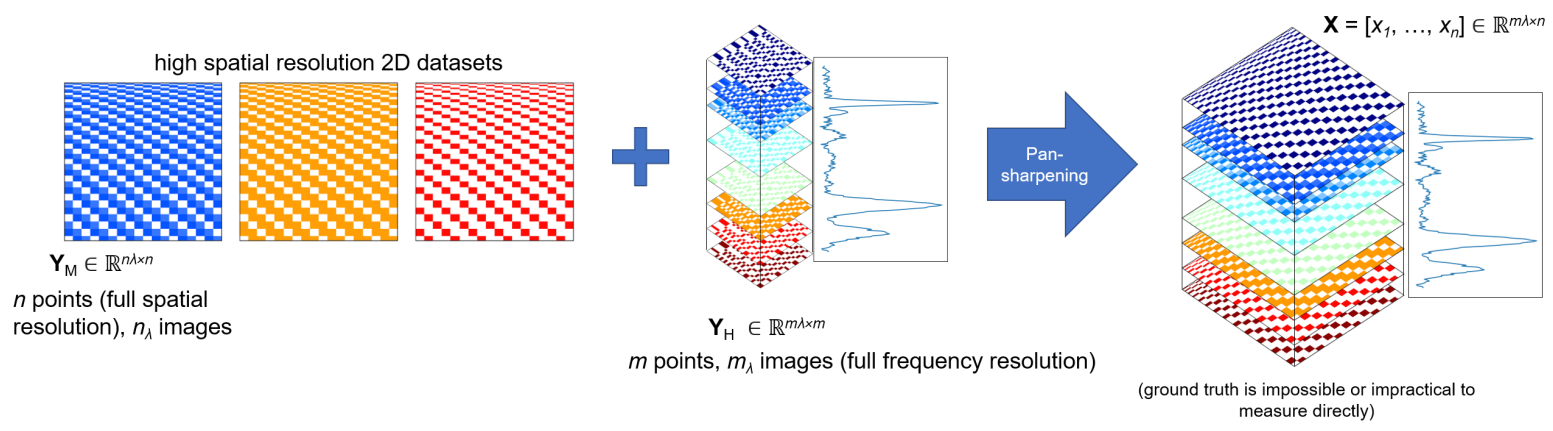

Figure 1. The overview of pan-sharpening algorithm: a series of high spatial resolution maps acquired at a given frequency and a low spatial resolution dataset with full frequency spectrum in each point can be combined to reconstruct a dataset with full spectral and full spatial resolution.
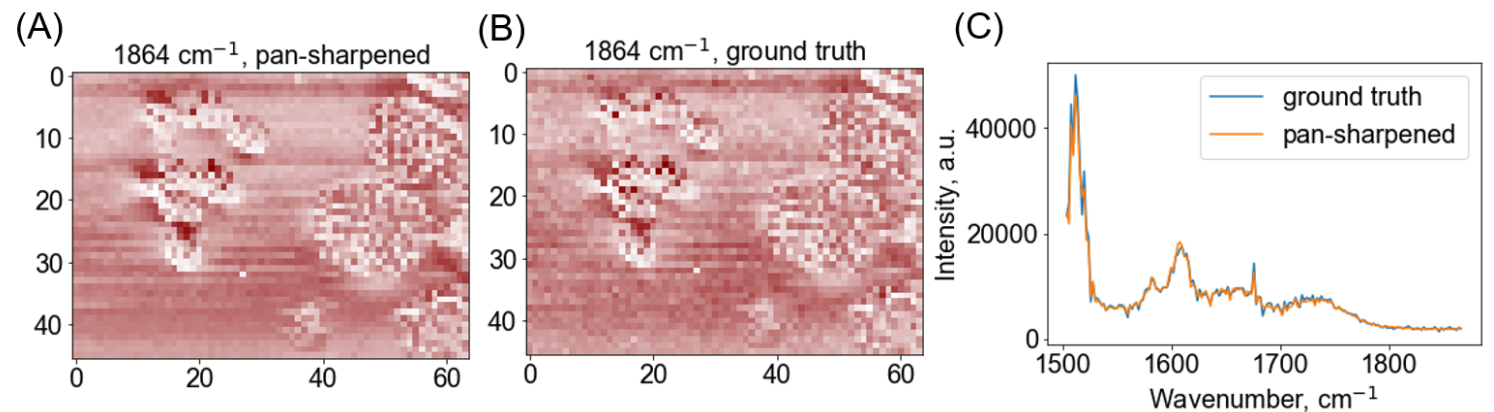

Figure 2. Pan-sharpened dataset is restored vey efficiently as evident from the comparison between single wavenumber maps (A, B) and point spectra (C). 